
allemande

51-2| 2019

Les Humanités environnementales : circulations et renouvellement des savoirs en France et en Allemagne

\title{
La certification Passivhaus entre Allemagne et France
}

Un modèle international de « construction durable » aux appropriations différenciées à l'échelle locale

\section{Marie Mangold}

\section{QpenEdition}

\section{Journals}

Édition électronique

URL : https://journals.openedition.org/allemagne/2022

DOI : 10.4000 /allemagne.2022

ISSN : 2605-7913

Éditeur

Société d'études allemandes

Édition imprimée

Date de publication : 10 décembre 2019

Pagination : 451-470

ISSN : 0035-0974

Référence électronique

Marie Mangold, «La certification Passivhaus entre Allemagne et France », Revue d'Allemagne et des pays de langue allemande [En ligne], 51-2 | 2019, mis en ligne le 02 décembre 2020, consulté le 19 mai 2021. URL : http://journals.openedition.org/allemagne/2022 ; DOI : https://doi.org/10.4000/ allemagne.2022 


\section{La certification Passivhaus entre Allemagne et France Un modèle international de «construction durable» aux appropriations différenciées à l'échelle locale}

\section{- Marie Mangold*}

\section{Introduction}

Cette contribution ${ }^{(1)}$ propose d'analyser la circulation des savoirs sur la «durabilité» entre Allemagne et France à l'aune du référentiel de construction Passivhaus. Apparu en Allemagne au début des années 1980, il se trouve en cours d'appropriation depuis la fin des années 2000 par les acteurs de la construction durable en France, avec l'organisation, entre 2007 et 2010, des Assises nationales de la construction passive à Grenoble puis du Salon Passi'bat à Paris. Le développement d'une fédération professionnelle de la construction passive depuis 2012 à Saverne, en Alsace, sera pris comme cas d'étude. Il s'agira en particulier d'analyser l'intégration du référentiel Passivhaus au sein de la production de la maison individuelle à l'échelle régionale.

Le contexte de diffusion du référentiel Passivhaus est celui du développement relativement récent des green buildings à l'échelle mondiale, qui marque la prise en compte des enjeux environnementaux dans le domaine du bâtiment. La structuration d'un marché de la construction durable découle de l'institutionnalisation de l'éco-construction ${ }^{(2)}$, intégrée progressivement au sein de politiques publiques

* Post-doctorante en sociologie, programme Interreg RES-TMO (2019-2021) «Solutions régionales pour des systèmes d'énergies renouvelables dans la Région métropolitaine trinationale du Rhin supérieur», laboratoire Sociétés, acteurs, gouvernement en Europe (SAGE, UMR 7363), CNRS et Université de Strasbourg, mmangold@unistra.fr.

1 Elle est issue d'une thèse en sociologie qui analyse plus largement les conditions de développement et de structuration d'un marché de la construction durable en Alsace entre 2012 et 2018: Marie MANGOLD, Pour une sociologie de la "maison durable»: entre production d'une offre techno-centrée et vécu des habitants, thèse (sociologie), Université de Strasbourg, 2018.

2 Sophie Némoz, «L'“éco-logis” politique: un dépaysement critique de l'habitat durable en Europe», Sciences de la société, 98 (2017), p. 31-43. 
environnementales et de logement. Cette institutionnalisation a suivi la logique top down du développement durable, en partant des instances internationales ${ }^{(3)}$ avant de s'appliquer aux politiques nationales ${ }^{(4)}$.

Concrètement, la prise en compte de l'environnement dans la construction emprunte une dynamique sélective, à partir d'une focale énergétique et techno-centrée ${ }^{(5)}$, et est marquée par des processus de certification conduisant à labelliser des bâtiments dits «exemplaires». En dynamisant l'industrie du bâtiment par le développement de procédés constructifs innovants qui visent l'obtention de nouvelles certifications, la construction durable s'intègre plus largement dans un marché de l'économie verte ${ }^{(6)}$ où les labels eux-mêmes se retrouvent en compétition.

Cette tendance se différencie nettement des initiatives en éco-construction des années 1970 en France, à l'exemple des habitants de maison solaire étudiés par Salvador Juan. Leur projet d'habitat comportait alors une dimension critique, « un refus du productivisme» ${ }^{(7)}$ qui visait en particulier le fonctionnement politique et l'économie capitaliste. Ceci laisse percevoir une évolution des rapports à la question environnementale et à la nature qui présente des points communs dans différents contextes nationaux, et notamment dans sa circulation entre Allemagne et France, à l'image des "passerelles franco-germaniques décelées dans la réalisation des projets de réhabilitation d'anciennes habitations rurales» et de la construction en pisé (terre crue) ${ }^{(8)}$.

Ma contribution est organisée en trois temps, partant du contexte d'émergence de la certification Passivhaus jusqu'à ses appropriations locales sur le terrain alsacien. Dans un premier temps, j'expliquerai comment le concept de «maison passive» a été développé en Allemagne dans les années 1990 et a progressivement été diffusé comme «modèle» de construction à l'échelle européenne et internationale. Je montrerai notamment que ce modèle, essentiellement basé sur des critères énergétiques, concurrence d'autres certifications qui intègrent des critères environnementaux plus globaux. L'exemple de la certification Haute Qualité Environnementale (HQE) en France est notamment significatif. Il s'agit ici de pointer la circulation de divers modèles de durabilité et la prédominance actuelle de l’approche énergétique dans différents contextes nationaux et climatiques.

3 La conférence onusienne Habitat I à Vancouver en 1976 marque d'abord une prise de conscience des processus d'urbanisation et de leurs impacts sociaux et environnementaux, puis, en 1996, la conférence «Sommet des villes» ou Habitat II, tenue à Istanbul, aboutit à l'adoption d'un plan stratégique, The Habitat Agenda, ratifié par 171 pays. L'habitat durable est dès lors inscrit sur l'agenda politique et se développe plus concrètement dans le cadre de politiques nationales. Philippe Hamman, Sociologie urbaine et développement durable, Bruxelles, De Boeck, 2012.

5 Christophe Beslay, Romain Gournet et Marie-Christine ZÉlem, «Le "bâtiment économe": utopie technicienne et "résistance" des usages", in: Jérôme Boissonade (dir.), La ville durable controversée. Les dynamiques urbaines dans le mouvement critique, Paris, Éditions Pétra, 2015, p. 335-363.

6 Marit Rosol, Vincent BÉAl et Samuel Mossner, "Greenest Cities? Urban Sustainability and the (Post-)Politics of Territorial Competitiveness ", Environment and Planning A, 49/8 (2017), p. 1710-1718.

7 Salvador JUAN, «Le temps et l'espace de la maison solaire», Espaces et sociétés, 46 (1985), p. 142.

8 Sophie Némoz, «Bâtir les humanités environnementales des matériaux géo-sourcés: construction et déconstruction des passerelles franco-germaniques", Revue d'Allemagne et des pays de langue allemande, 50/2 (2018), p. 377-392, ici p. 387. 
Puis, dans un second temps, le marché alsacien de la construction passive sera pris comme cas d'étude. J'expliciterai d'abord les jeux d'acteurs et la structuration en cours d'une offre de maison passive dont j'ai observé le développement lors de salons régionaux de l'habitat de 2013 à 2018. Les acteurs à l'initiative de cette offre adoptent un discours critique à l'égard des réglementations françaises et se positionnent comme «pionniers» d'une «construction durable» basée sur des modèles internationaux.

Enfin, dans un troisième temps, des controverses entre acteurs locaux de la construction passive seront exposées. Je mettrai en exergue le flou réglementaire autour de ce modèle de construction, observable dans les appropriations variables qu'en font les maîtres d'œuvre et constructeurs, à travers les questions de labellisation et d'équipements énergétiques de chauffage dans une maison annoncée comme "sans chauffage». La maison passive se présente alors comme un modèle en débat, faisant l'objet d'appropriations diversifiées dans le discours des maîtres d'œuvre et constructeurs $^{(9)}$.

\section{Dispositif méthodologique}

Sur le plan méthodologique, le raisonnement s'appuie sur des enquêtes de terrain ${ }^{(10)}$, conduites de 2012 à 2018 en Alsace ${ }^{(11)}$, fondées sur différents matériaux : 44 séquences d'observations menées lors de différents événements liés au «logement durable»: salons de l'habitat régionaux (Colmar et Strasbourg, de 2013 à 2018), conférences portant sur les bâtiments performants ou encore les matériaux biosourcés, visites de quartiers «durables» allemands (Fribourg, Tübingen), observations de groupes d'habitat participatif, portes ouvertes de constructeurs, etc.

Un corpus de revues spécialisées a également permis de suivre les évolutions du domaine sur la période couverte: offre immobilière locale (suivie notamment via le magazine LogicImmo) et nationale (revue en ligne Batiactu, destinée aux acteurs de la construction), études et publications du Commissariat général au développement durable.

Enfin, un corpus de 65 entretiens a été constitué, dont 7 entretiens approfondis avec des maîtres d'œuvre ou constructeurs investis dans la construction durable en Alsace, que je mobiliserai particulièrement ici.

9 Constructeur et maître d'œuvre sont à distinguer: un constructeur propose une offre de construction globale, via un contrat CCMI (contrat de construction de maison individuelle), en s'associant à des entreprises partenaires, tandis qu'un maître d'œuvre assure la conception de la maison et le suivi du chantier, mais le client reste libre de choisir les entreprises partenaires. Un architecte, par exemple, peut assurer cette maîtrise d'œuvre; cependant, tous les maîtres d'œuvre ne sont pas architectes.

10 Ces enquêtes ont été menées dans le cadre d'une thèse de doctorat en sociologie (2012-2018) qui a pris place au sein du projet de recherche collectif CIMBEES (Conception et industrialisation de modules de bâtiment à haute qualité environnementale et sociale, FUI-Oséo-Feder, 14 e AAP, 2012-2015), porté par le laboratoire SAGE (Sociétés, Acteurs, Gouvernement en Europe), sous la direction de Philippe Hamman.

11 Ces enquêtes de terrain ont été initiées avant le redécoupage régional de 2015. 


\section{La diffusion du référentiel Passivhaus, une illustration de la focale énergétique de la construction durable}

Le contexte même d'émergence de ce standard allemand «témoigne de l'importante influence de l'internationalisation des processus et acteurs entrepreneurs dans la percolation des innovations au sein des politiques locales " ${ }^{(12)}$. Il est en effet issu d'une collaboration entre deux scientifiques spécialistes de la construction, Bo Adamson, professeur à l'Université de Lund en Suède (Institute of Technology, Department of Building Science), et Wolfgang Feist, physicien allemand à Darmstadt (Institut für Wohnen und Umwelt $)^{(13)}$. Les deux experts se sont notamment inspirés de maisons à faible consommation énergétique ${ }^{(14)}$ en Scandinavie et en Amérique du Nord, en renforçant la performance énergétique du bâtiment grâce à des recherches sur le comportement thermique des murs, isolants et fenêtres. Comme le soulève Julie Neuwels, «l'apport des politiques publiques fut fondamental dans l'émergence de cette innovation et son implémentation dans divers pays européens " ${ }^{(15)}$. Le Land de Hesse accueille en effet favorablement et soutient financièrement la construction des premières maisons dites "passives». Quatre maisons Passivhaus sont construites en 1991 à Darmstadt-Kranichstein, en ayant recours à des matériaux existants (murs en pierre, fenêtres en bois) mais également à des innovations telles que le premier modèle de fenêtre triple vitrage ou encore une ventilation plus efficace pour éviter les déperditions énergétiques.

À la suite de ces premières expérimentations, Wolfgang Feist et ses collaborateurs fondent le Passivhaus Institut (PHI) en 1996, qui devient l'organisme de référence du bâtiment passif, en proposant la certification Passivhaus à la fois pour les bâtiments et pour certains matériaux et composants spécifiques (VMC double-flux ${ }^{(16)}$, fenêtres...). Le PHI développe conjointement un logiciel de calcul, le PassivhausProjektierungspaket (PHPP), rendu publiquement disponible en 2004. Ce logiciel est utilisé par les acteurs de la construction passive dans d'autres pays et ce même lorsque les bâtiments ne sont pas certifiés par le PHI; j'y reviendrai. Les préconisations techniques de construction sont fondées sur des calculs, et la mesure des résultats, une fois le bâtiment construit, permet d'attester l'atteinte du standard "passif» à partir de

12 Julie Neuwels, "Construction durable: expertise et contre-expertise d'architectes», VertigO, 13/2 (2013), p. 9, en ligne: vertigo.revues.org/14166.

13 Les informations concernant l'historique du concept Passivhaus sont tirées des sites Internet suivants: antenne française du Passivhaus Institut: www.lamaisonpassive.fr/darmstadt-25-ans-apres-toujoursvaillant/; entretien avec Wolfgang Feist: www.construction21.org/france/articles/fr/la-premieremaison-passive-entretien-avec-dr-wolfgang-feist-2-2.html; site allemand du Passivhaus Institut: passiv.de/.

14 En ce sens, la création d'un standard Passivhaus ne fait qu'institutionnaliser et standardiser une recherche d'autonomie énergétique déjà en cours depuis les années 1970, dans un mouvement de «contre-culture» en architecture. Fanny Lopez donne l'exemple d'Alexander Piker, architecte anglais ayant développé un concept de maison autonome (1971-1979) qui utilise notamment les principes du solaire passif, et dont le projet a bénéficié d'un appui institutionnel et industriel. Voir Fanny Lopez, Le rêve d'une déconnexion. De la maison autonome à la cité auto-énergétique, Paris, Éditions de la Villette, 2014.

15 J. Neuwels, «Construction durable» (note 12).

16 La VMC double-flux autorégule le renouvellement de l'air et permet de préchauffer l'air entrant à l'aide de l'air sortant grâce à un échange de calories, et vice-versa selon les saisons. 
seuils à respecter. En effet le référentiel de construction passive se base sur trois types d'exigences chiffrées de résultats: une efficacité énergétique du bâtiment (besoins en chauffage limités à $15 \mathrm{kWh} / \mathrm{m}^{2}$.an), une qualité constructive (limitation des flux d'air non maîtrisés) ainsi qu'une faible empreinte énergétique tous usages confondus ${ }^{(17)}$.

Si cette initiative de rationalisation et de standardisation de l'éco-construction est précurseuse dans les années 1990, elle correspond aujourd'hui à l'approche mainstream de la construction durable: des projets et certifications priorisant des critères énergétiques, au détriment d'une approche environnementale plus globale, conçus par des techniciens et ingénieurs qui favorisent un solutionnement technique des questions environnementales à partir de leurs expertises ${ }^{(18)}$, et impulsés par des politiques d'aménagement à l'échelle locale. Les exemples suivants illustrent cette tendance générale et témoignent de la circulation concurrentielle entre différents modèles de durabilité.

Depuis plusieurs années, la construction passive constitue une référence de bâtiments dits "exemplaires" en matière d'efficacité énergétique. Ses objectifs restent supérieurs aux réglementations énergétiques nationales progressivement imposées dans le bâtiment. En Allemagne par exemple, la construction passive ne constitue pas la référence réglementaire de la construction neuve, dont les objectifs sont comparables aux réglementations thermiques françaises actuelles ${ }^{(19)}$. En réaction à la directive européenne de 2010 indiquant un objectif de Nearly Zero Energy Building (NZEB) à l'horizon 2020, le Conseil allemand de la construction durable (Deutsche Gesellschaft für Nachhaltiges Bauen, DGNB) ${ }^{(20)}$ a développé sa propre certification pour construire des bâtiments au bilan carbone «neutre», tout en prenant en compte d'autres facteurs environnementaux tels que le cycle de vie du bâtiment ${ }^{(21)}$. Parallèlement à ces réglementations et certifications, qui évoluent régulièrement et sont parfois difficiles à saisir dans leur différenciation pour le maître d'ouvrage ${ }^{(22)}$, la construction Passivhaus a fait l'objet de nombreuses réalisations. D’après la base de données internationale du PHI, il y aurait 2334 bâtiments passifs en Allemagne ${ }^{(23)}$ et 4547 à l'échelle mondiale. Ce chiffre ne donne cependant qu'une indication minimale de l'état de la construction passive, car cette dernière s'est parfois affranchie de la référence au PHI dans d'autres contextes nationaux.

17 Soit une consommation maximum d'énergie primaire limitée à $120 \mathrm{kWh} / \mathrm{m}^{2}$.an pour la totalité des usages effectués dans le bâtiment, une fois celui-ci équipé (électroménager, audiovisuel, etc.) par l'habitant.

18 Pierre Lascoumes, L'éco-pouvoir: environnements et politiques, Paris, La Découverte, 1994.

19 Sven Rösner et Marie Boyette, «Allemagne: le bâtiment et l'énergie en chiffres ", Annales des Mines, 90 (2018), p. 12-16. Selon les auteurs, «la réglementation actuelle (EnEV 2014-2016) fixe une consommation maximale qui est en moyenne de $56 \mathrm{kWh} / \mathrm{m}^{2}$.an pour le chauffage, l'eau chaude, la climatisation et la ventilation» (p. 12). En France, la RT 2012 fixe ce seuil à $50 \mathrm{kWh} / \mathrm{m}^{2}$.an.

20 www.dgnb-system.de/en/.

21 Le cycle de vie prend en compte toutes les activités qui entrent en jeu dans la fabrication, l'utilisation, le transport et la destruction du bâtiment. Voir www.actu-environnement.com/.

22 Le maître d'ouvrage est à l'origine de la commande d'un projet de construction, tandis que le maître d'œuvre en est l'exécutant.

23 Dont seulement 492 sont certifiés par le PHI, les autres étant considérés comme passifs car construits par des maîtres d'œuvre ou constructeurs ayant validé la formation du PHI. Voir passivhausprojekte.de. 
Hors Allemagne, ce standard fait modèle et témoigne de l'exportation d'un cadre de construction durable développé à l'échelle locale vers d'autres pays et contextes climatiques et énergétiques. Il y fait l'objet d'appropriations qui visent à redéfinir ses objectifs tout en s'appuyant sur ses principes de base et ses logiciels de calcul. On le voit s'intégrer au sein de cadres réglementaires locaux, par exemple dans la région de Bruxelles-Capitale, où le standard passif, dans une version assouplie, a été imposé en janvier 2015 pour toute constructive neuve ${ }^{(24)}$. Il en est de même au Luxembourg, où la construction d'une maison neuve doit se conformer aux normes de la maison passive depuis janvier 2017, tout en admettant un seuil de consommation énergétique en chauffage un peu plus élevé( ${ }^{(25)}$. Plusieurs pays disposent d'ailleurs de leur propre antenne nationale de construction passive, comme c'est le cas en Belgique, en Suisse (qui se présente comme un complément ou une alternative au standard Minergie- $P$ ) ${ }^{(26)}$, en Espagne, au Royaume-Uni mais également aux États-Unis ${ }^{(27)}$.

L'importation du standard Passivhaus à l'échelle européenne et internationale introduit une concurrence avec d'autres certifications environnementales pré-existantes. On peut prendre l'exemple de la certification BREEAM (Building Research Establishment Environmental Assessment Methodology), pionnière pour la qualité environnementale de la construction ${ }^{(28)}$, qui a été développée au Royaume-Uni en 1990. Dans une logique de «carbonisation» du développement durable ${ }^{(29)}$ et de focale sur le changement climatique, la construction à haute performance énergétique, incarnée notamment par la construction passive, semble cependant être progressivement priorisée. La construction durable se tourne vers un modèle de construction neutre en carbone, comme l'explique Jenny Pickerill:

«In Britain, eco-housing has become synonymous with low or zero carbon housing. Although the early roots of eco-homes were diverse (and included concerns for

24 www.lamaisonpassive.fr/le-passif-desormais-obligatoire-en-region-de-bruxelles-capitale/. Voir également les travaux de Julie Neuwels sur les controverses suscitées par cette imposition réglementaire du passif au sein du domaine du bâtiment et notamment entre les architectes: Julie NeuweLs, «Politique de performance énergétique des logements à Bruxelles: une logique industrielle structurellement inégalitaire», Espaces et sociétés, 170 (2017), p. 73-90; id., «Construction durable» (note 12).

25 Soit $22 \mathrm{kWh} / \mathrm{m}^{2}$. an contre 15 pour le standard du PHI. Voir «Les maisons passives en pleine action», Le Quotidien (Luxembourg), 22 août 2018; www.myenergy.lu/fr/accueil.

26 Minergie est une association fondée en 1998 à l'initiative des cantons de Berne et de Zurich: «Le concept visait originellement une bonne performance énergétique du bâtiment à coût maîtrisé (Minergie-P pour "passif" lancé en 2002) et a évolué progressivement vers une amélioration de la qualité de vie et la diminution des atteintes causées à l’environnement (Minergie-Éco pour “écologie” et Minergie-P-Éco pour "passif et écologique”).» Voir Lionel CAUCHARD, Les collèges d'experts et la fabrique de la normalisation technique. Hybridation normative et performation de la Haute Qualité Environnementale (HQE) des Bâtiments en France, thèse (sociologie), Université Paris-Est, 2010, p. 387.

27 Plateforme Maison passive en Belgique: www.maisonpassive.be/spip.php; la Maison passive suisse: ig-passivhaus.ch/fr/ig-passivhaus-home; Plataforma Edificación Passivhaus en Espagne: www.plataforma-pep.org/; Passivhaus Trust au Royaume Uni: www.passivhaustrust.org.uk/; Passivhaus Institut US aux États-Unis: www.phius.org/home-page.

28 Elle prend en compte 10 critères, auxquels sont attribués des points: Gestion des opérations, Santé et bien-être, Énergie, Transport, Eau, Matériaux, Déchets, Terrains utilisés et écologie, Pollution, Innovation. Voir www.breeam.com/. 
biodiversity, materials scarcity, pollution, water-use reduction), reducing carbon became the dominant discourse that framed the need for, and function of, eco-housing» ${ }^{(30)}$.

Le cas de la France illustre particulièrement bien cette concurrence entre différents modèles de construction durable. Le contexte français est d'abord marqué par une tentative de généralisation de la démarche française Haute Qualité Environnementale, créée en 1996 sous l'impulsion du Plan Urbanisme Construction Architecture (PUCA). L'association HQE se donne pour objectif de "promouvoir, dans une perspective de développement durable, l'amélioration de la qualité environnementale du cadre de vie bâti » ${ }^{(31)}$. Elle propose un cadre de référence, la "démarche HQE», organisée en 14 cibles $^{(32)}$, permettant de définir la qualité environnementale des logements, via quatre volets: l'éco-construction, l'éco-gestion, le confort et la santé. En 2004, l'association HQE est reconnue d'utilité publique et lance la certification $N F H Q E^{\mathrm{rm}}$, en collaboration avec l'organisme certificateur AFNOR ${ }^{(33)}$. Au-delà d'une performance énergétique, «la qualité environnementale consiste à maîtriser les impacts sur l'environnement extérieur et à créer un espace intérieur sain et confortable ${ }^{(34)}$.

L'approche environnementale de la HQE s'est néanmoins vue détrônée par la focale énergétique des politiques environnementales, orientée, comme on l'a vu, par des directives européennes sur le Nearly Zero Energy Building. Cette orientation se concrétise dans la production de nouvelles réglementations thermiques françaises, la RT 2012 et la RT $2020^{(35)}$, issues des lois Grenelle I et II (2009-2010). En France, c'est en effet à partir du Grenelle de l'environnement ${ }^{(36)}$ que la focale climatique et énergétique est pleinement observée ${ }^{(37)}$. Un cadrage technique ${ }^{(38)}$ centré sur les questions énergétiques

30 Jenny Pickerill, Eco-Homes. People, Place and Politics, London, Zed Books, 2016, p. 18. «En GrandeBretagne, l'éco-construction est devenue synonyme de construction à faible voire zéro émission de carbone. Bien qu'à l'origine l'habitat écologique recouvre des préoccupations diverses (ce qui incluait les questions relatives à la biodiversité, à la disponibilité des matériaux, à la pollution et à la réduction de la consommation d'eau), la baisse des émissions carbone est devenue l'objectif dominant, qui définit le besoin et les fonctions premières de l'éco-construction " (ma traduction).

31 www.hqegbc.org/wp-content/uploads/doc/hqe-statuts.pdf.

32 À savoir: 1. Relation harmonieuse des bâtiments avec leur environnement immédiat, 2. Choix intégré des procédés et des produits de construction, 3. Chantier à faible nuisance, 4. Gestion de l'énergie, 5. Gestion de l'eau, 6. Gestion des déchets d'activités, 7. Gestion de l'entretien et de la maintenance, 8. Confort hygrothermique, 9. Confort acoustique, 10. Confort visuel, 11. Confort olfactif, 12. Conditions sanitaires des espaces, 13. Qualité de l'air, 14. Qualité de l'eau. Notons cependant qu'aujourd'hui, la démarche HQE propose un «cadre de référence» global, élargissant les quatorze cibles de départ.

33 Association française de normalisation.

34 Voir Guy Chautard, «Développement durable et construction», in: Bertrand Zuindeau (dir.), Développement durable et territoire, Villeneuve d'Ascq, Presses universitaires du Septentrion, 2010, p. 388.

35 La RT 2020 fixe l'objectif de construire en bâtiment à énergie positive (Bepos) d'ici 2020.

36 Processus de concertation ciblant certains domaines impactant l'environnement, lancé en 2007 par le gouvernement français.

37 Vincent BÉAL, «Le déclin du développement durable. Changement climatique et transformation de la gouvernance environnementale dans les villes françaises et britanniques », in: Isabelle HaJEK et Philippe Hamman (dir.), La gouvernance de la ville durable entre déclin et réinventions. Une comparaison Nord/Sud, Rennes, Presses universitaires de Rennes, 2014, p. 96.

38 C'est-à-dire un débat sur les moyens techniques à mettre en place et non sur la nature même du changement. 
est opéré dès le départ, par des groupes de travail accordant une place primordiale aux acteurs économiques ${ }^{(39)}$. Parallèlement à la production de ces réglementations, de nouvelles certifications voient le jour au niveau national. Par exemple, pour la construction neuve, le label $B B C$-Effinergie ${ }^{(40)}$, défini à partir du référentiel de l'association Effinergie $^{(41)}$, anticipe la RT $2012^{(42)}$. Puis, sont apparus d'autres labels tels que BBC-Effinergie+ ou Bepos Effinergie 2013 ${ }^{(43)}$, afin d'attester une performance énergétique supérieure. Au courant de l'année 2017, l'expérimentation «Énergie+ Carbone-», initiée par l'État en novembre 2016 et qui anticipe la RT 2020, a conduit au lancement de plusieurs labels: E+C-, BBC Effinergie 2017, Bepos Effinergie 2017 et Bepos+ Effinergie 2017. D'autres certifications existent également, comme le label Bâtiment Bas Carbone de l'association BBCA $^{(44)}$, entré en vigueur en mars 2016, et qui se base sur l'empreinte carbone d'un bâtiment. On observe donc une multiplication des labels et une évolution des critères d'obtention de ceux-ci, devenant toujours plus exigeants. De la sorte, c'est également une échelle de mesure objectivée qui se diffuse et retraduit le registre pluriel de la durabilité suivant une lecture technique organisée d'après un échelonnement de performances. De façon comparable, la certification Passivhaus peut désormais être qualifiée de "plus» ou de "premium» en cas de bâtiment passif à énergie positive, c'est-à-dire intégrant une production d'énergie renouvelable ${ }^{(45)}$.

Le cadre réglementaire français et les certifications encouragées par l'action publique $^{(46)}$ ne se réfèrent toutefois pas à la construction passive. Ce sont dès lors les acteurs du secteur de la construction, et notamment les entreprises locales du bâtiment, qui se sont emparés du modèle d'outre-Rhin pour proposer une offre de construction passive dont je vais à présent expliciter les jeux d'acteurs et controverses à partir du cas de la construction de maisons individuelles en Alsace.

\section{Jeux d'acteurs et structuration du marché alsacien de la construction passive}

Le PHI de Darmstadt est officiellement représenté en France par la Maison Passive France $(\mathrm{MPF})^{(47)}$. Cette association fédère des professionnels du bâtiment issus

39 Julie Pollard, «Les effets du Grenelle de l'environnement: l'énergie dans le secteur du bâtiment», in: Daniel Boy, Mathieu Brugidou, Charlotte Halpern et Pierre Lascoumes (dir.), Le Grenelle de l'environnement. Acteurs, discours, effets, Paris, Armand Colin, 2012, p. 215-235.

$40 B B C$ pour bâtiment basse consommation.

41 Effinergie est une association créée en 2006 ayant pour objectif de départ la promotion des $B B C$, qui ont servi de modèle à l'élaboration de la RT 2012. D'après Lionel Cauchard, la certification BBC-Effinergie a été soutenue par le Centre scientifique et technique du bâtiment (CSTB: organisme public certificateur des produits de construction) face à la concurrence de la certification suisse Minergie: L. CAUChARD, Les collèges d'experts (note 26), p. 291.

42 La RT 2012 a été mise en application dans le secteur résidentiel de la construction neuve au $1^{\text {er }}$ janvier 2013, rendant caduque le recours au label BBC dans la construction neuve.

43 Bepos pour bâtiment à énergie positive.

44 Association pour le développement du Bâtiment bas carbone.

45 www.lamaisonpassive.fr/wp-content/uploads/2016/12/CriteresDeCertification.pdf.

46 Henri Bergeron, Patrick Castel et Sophie Dubuisson-Quellier, «Gouverner par les labels. Une comparaison des politiques de l'obésité et de la consommation durable», Gouvernement et action publique, 3 (2014), p. 7-31. 
de différents corps de métier, dont la maîtrise d'œuvre, mais également des maîtres d'ouvrage publics et privés. Elle constitue un réseau autour de la construction passive et organise des événements collectifs tels que le Salon Passi'bat, dont la onzième édition a eu lieu en mars 2018 à Paris ${ }^{(48)}$.

À l'échelle locale, mes enquêtes de terrain - en particulier les observations menées chaque année de 2013 à 2018 aux salons de l'habitat en Alsace - ont mis en évidence un certain engouement pour la construction de maisons passives. Ce dernier se traduit par le développement conséquent d'une offre de constructeurs et de maîtres d'œuvre dans ce créneau. La Fédération française de la construction passive (FFCP), association faisant la promotion du bâtiment passif en organisant notamment des formations mensuelles destinées aux professionnels du bâtiment ${ }^{(49)}$, est apparue comme un acteur central dans l'impulsion de cette dynamique à l'échelle locale. Elle a été créée en 2012 par d'anciens administrateurs de la $\mathrm{MPF}^{(50)}$, suite à un conflit au sein du collectif, et est présente au Salon de l'habitat de Colmar depuis 2014. La FFCP propose sa propre certification passive, en partie basée sur des calculs réalisés à partir du logiciel PHPP (Passive House Planning Package ou Passivhaus-Projektierungspaket), alors que la MPF fait toujours transiter les dossiers de certification par le PHI de Darmstadt. S'affichant comme organisme national de la construction passive, la FFCP est basée à Saverne en Alsace et est de facto particulièrement active dans la région ${ }^{(51)}$. Elle soutient à la fois la construction de bâtiments passifs collectifs, relevant du secteur tertiaire ou du logement, et fédère également de nombreux constructeurs et maîtres d'œuvre de maisons individuelles.

J'ai pu observer au fur et à mesure des années de l'enquête l'accroissement progressif du pôle de la FFCP ainsi qu'une communication accrue autour de la construction passive au sein des salons de l'habitat de Colmar et de Strasbourg (voir encadré et fig. 1). On repère ainsi toute une dynamique d'adaptation des constructeurs et maîtres d'œuvre à ce nouveau référentiel, en même temps que leur investissement participe en retour à la (re)définition de la construction passive. Précisément, dans ce processus de développement de la construction passive, cette dernière est assimilée et légitimée comme modèle de la "construction durable», comme on peut le voir dans le récent ouvrage Habitat durable: l'évidence de la construction passive ${ }^{(52)}$, co-écrit par le président de la FFCP, et dans les discours que je déplie ci-dessous, faisant des constructeurs de maison passive des «pionniers» autoproclamés de la «construction durable» en France.

48 www.passibat.fr/.

49 La FFCP, dont le président est Jean-Claude Tremsal, est étroitement liée à une entreprise de formation gérée par ce dernier, nommée Oze (Objectif zéro énergie), permettant aux adhérents de la Fédération de se former à la construction passive: fedepassif.fr/; passivhaus.fr/.

50 On note qu'auparavant, quatre éditions des Assises nationales de la construction passive ont eu lieu de 2007 à 2010, soutenues par la MPF. D’après Jean-Loup Bertez, cofondateur de la FFCP avec Jean-Claude Tremsal et organisateur de ces Assises, la FFCP est inscrite dans leur continuité : «Au bout de quatre ans, on ne voulait plus faire des choses à part mais faire rentrer la construction passive dans le monde du bâtiment en général, c'est pourquoi on a fondé la Fédération» (Salon énergie-habitat de Colmar, 2017).

51 Les adhérents sont situés dans toute la France mais plus nombreux dans le Grand Est. Voir: fedepassif. fr/les-adherents/.

52 Jean-Loup Bertez et Jean-Claude Tremsal, Habitat durable: l'évidence de la construction passive, Paris, Gallimard, 2017. 


\section{La FFCP, acteur central du Salon de l'habitat de Colmar, et la MPF à Strasbourg}

Le pôle FFCP du Salon énergie-habitat de Colmar de 2014 - première édition où elle est présente - comprend 10 exposants dont quatre maîtres d'œuvre/architectes. Le président de la FFCP est programmé dans les conférences du salon en proposant des interventions telles que "Construire passif aujourd'hui c'est possible» ou «Voir et comprendre le fonctionnement d'une maison passive». À partir de 2015, les conférences liées à la FFCP sont détaillées dans un programme à part, en distinction des autres conférences du salon, et font à présent intervenir différents types de professionnels (maître d'œuvre, entreprise de fenêtres, de matériaux, etc.). Le dépliant du salon consacre une pleine page à la construction passive, stipulant que « [le salon] Énergie Habitat se tourne définitivement vers des solutions de confort, d'économie et d'innovation pour votre habitat en consacrant un pôle entier à la FFCP ».

Les années suivantes, le salon propose toujours des conférences sur le fonctionnement global et le principe de la maison passive, mais également des interventions plus pointues sur le plan technique, sur des questions de ventilation, de choix de fenêtres, de matériaux ("Système d'isolation sous dalle certifié passif», en 2016) ou encore de techniques de production d'énergie ("Solar Water box», en 2016). Il s'agit désormais d'un événement à part entière hébergé dans le Salon de l'habitat, la FFCP proposant sur les quatre jours du salon deux journées "pros» (vendredi et lundi) ${ }^{(1)}$ et deux journées "grand public» (samedi et dimanche).

En 2017 et 2018, le salon fait référence à un «pôle passif», avec notamment un espace dédié à la présentation d'une «mini maison passive», c'est-à-dire l'exposition de morceaux de murs (comprenant différents systèmes et matériaux: bois avec isolant naturel ou béton cellulaire, etc.), d'une VMC double flux ou encore de fenêtres triple vitrage. Ce démonstrateur est installé au centre du pôle et donne aux visiteurs la possibilité concrète de visualiser concrètement en quoi consiste une maison passive.

On note que, parallèlement à ces évolutions, entre 2014 et 2018, le pôle FFCP s'est également considérablement agrandi, passant de 10 exposants à 50 lors du dernier Salon de I'habitat enquêté ${ }^{(2)}$, bénéficiant dès lors de son propre espace de conférences. Une salariée a été recrutée afin de gérer les différents événements organisés par la FFCP, dont ce Salon de l'habitat ${ }^{(3)}$. Enfin, en 2018 , la FFCP se diversifie en proposant un projet baptisé OSCAR (Organiser, Sécuriser, Contrôler, Accompagner, Rénovation énergétique), basé non pas sur la construction passive mais sur la rénovation énergétique.

L'engouement pour la maison passive au Salon de l'habitat de Strasbourg est moins flagrant, ce qui n'est peut-être pas sans lien avec le fait que ce salon soit ciblé sur la construction en bois et non les questions énergétiques. En 2013, le président de la FFCP donne deux conférences sur la maison passive, puis il focalisera son action sur le salon de Colmar. Les années suivantes, on repère quelques conférences sur la maison passive, intitulées «En route vers la maison passive», ou encore "La maison passive: principe et performances» aux salons de Strasbourg de 2015, 2017 et 2018. Elles sont toutes données par le même intervenant, un maître d'œuvre de maison en bois ayant passé le diplôme du PHI en 2014 et représentant l'organisme MPF, et non la FFCP, au salon.

1 II s'agit de journées payantes avec un programme spécifique, et comprenant un repas entre professionnels.

2 Tous les exposants prévus n'étaient cependant pas réellement présents en 2018. Notons qu'en 2017 nous avions déjà noté 41 exposants alors qu'ils n'étaient que 26 en 2016.

3 Une architecte est également chargée de vérifier les aspects réglementaires des bâtiments à certifier. 
Fig. 1 : Pôle FFCP avec «mini maison passive» : systèmes constructifs, isolants, fenêtres, VMC double flux

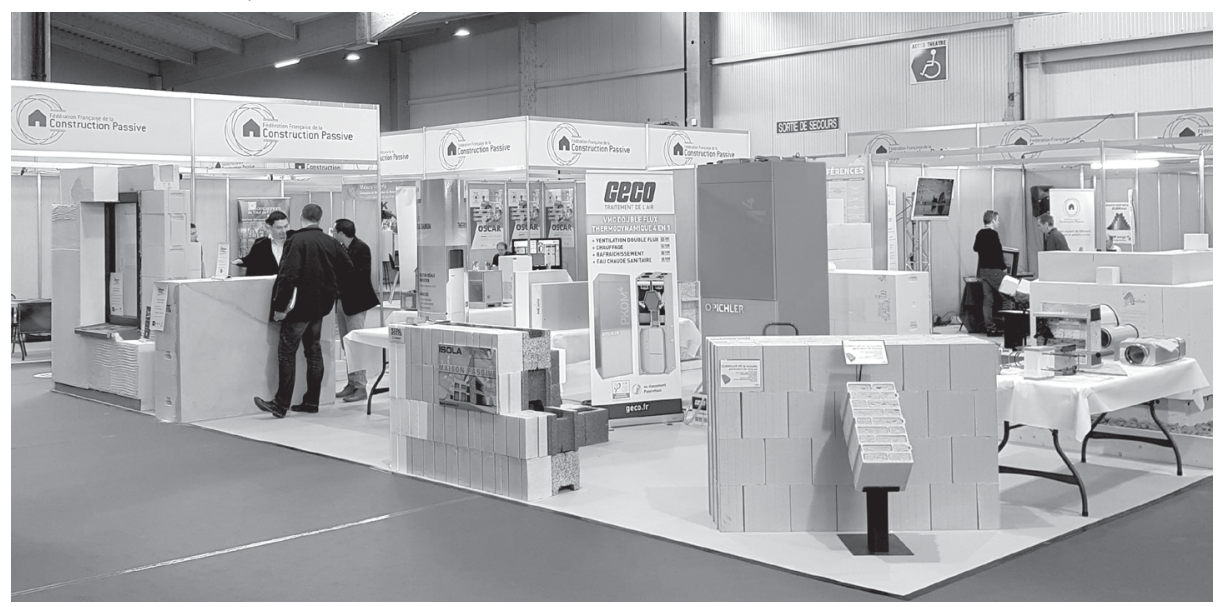

Marie Mangold, Salon de l'habitat de Colmar, 2018.

Il est intéressant de noter que les constructeurs et maîtres d'œuvre adhérant à la FFCP sont généralement des entreprises de maisons individuelles existant de longue date et qui ont pris le "virage» de la construction passive. Par exemple, le maître d'œuvre Éco-passive était auparavant connu sous le nom de Construction Voltz, entreprise de gros œuvre de maisons en béton. Il construit aujourd'hui des maisons passives et "écologiques" en béton cellulaire (voir fig. 2), en s'associant parfois à un architecte ayant lui aussi pris un nom d'entreprise faisant référence à la construction passive: Alsace maison passive. De façon similaire, l'entreprise $M a c c-3$, entreprise de gros œuvre proposant un système de murs en béton cellulaire, s'est lancée dans la maîtrise d'œuvre complète et la maison passive en créant l'entreprise Innovalys.

On assiste donc à des formes de reconfiguration de ces entreprises dont l'outil publicitaire (changement de nom et d'axes de communication) est essentiel pour la vente de leurs prestations, et qui cherchent à se différencier, dans un marché très concurrentiel, par

Fig. 2 : Un maître d'œuvre de maisons en béton se renouvelant dans la construction passive

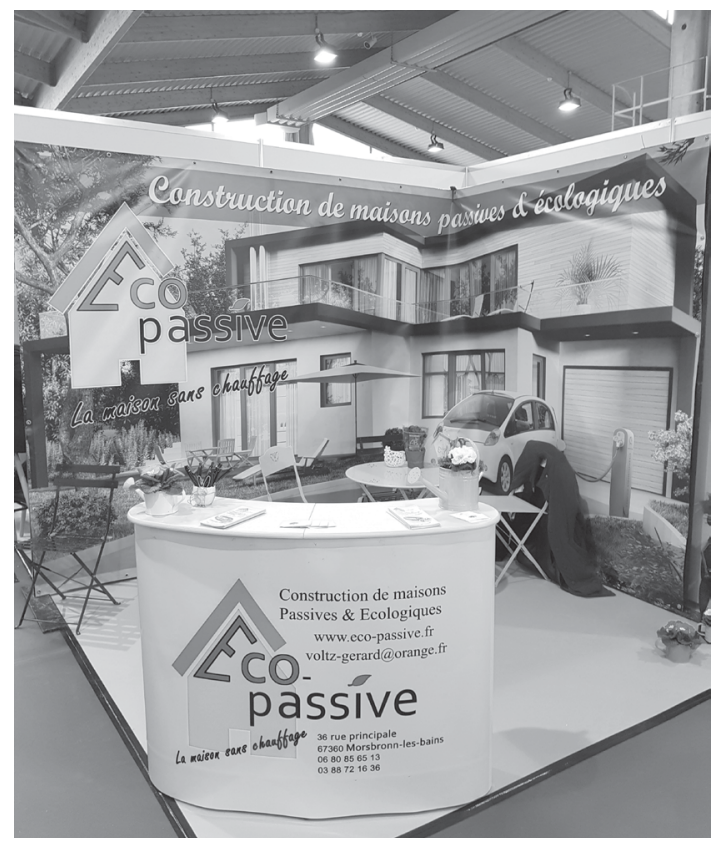

Marie Mangold, Salon de l'habitat de Colmar, 2018 
leur inscription dans le référentiel de la construction passive. Pour certaines d'entre elles, il s'est agi de faire évoluer leur offre de maison à haute performance énergétique, de la maison basse consommation (BBC) à la maison passive. Par exemple, l'entreprise Avenir \& Bois proposait encore en 2013 des maisons BBC. Puis, leur communication a évolué vers la maison passive, le cogérant d'Avenir \& Bois ayant notamment été diplômé du PHI en 2014, l'autorisant ainsi à se déclarer officiellement concepteur certifié PHI de maison passive. On retrouve par ailleurs cette évolution marquée en observant les publicités du magazine immobilier Logic-Immo Bas-Rhin ${ }^{(53)}$ : à partir du numéro de juillet 2014, l'encart publicitaire d'Avenir \& Bois se voit ajouter la mention «fabricant labellisé de maisons passives», et, en septembre 2014, l'entreprise organise des journées portes ouvertes comprenant des «conférences sur la RT 2012 et la maison passive» ${ }^{(54)}$.

Outre les conférences proposées aux salons de l'habitat ainsi que les exemples de systèmes constructifs ou d'équipements exposés, par exemple, dans l'espace «mini maison passive» au Salon de l'habitat de Colmar, les maîtres d'œuvre et constructeurs alsaciens enquêtés proposent à la clientèle de visiter leurs maisons passives lors de portes ouvertes. Ces portes ouvertes peuvent être l'objet de conférences de présentation du principe, des enjeux et des intérêts de la construction passive. On retient donc qu'en Alsace l'offre de maison passive s'est structurée et étendue ces dernières années et a intégré les canaux de communication du marché de la maison individuelle (salons de l'habitat, magazines immobiliers, portes ouvertes...).

Face à cette structuration d'une offre de construction passive, on peut se demander pourquoi les acteurs favorisent ce référentiel plutôt que l'objectif affiché par les politiques françaises de bâtiment Bepos. Dans leurs discours, les enquêtés évoquent l'aspect qualitatif et fiable, en termes de résultats énergétiques mesurables, de la construction passive. Ils adoptent une posture critique vis-à-vis des référentiels de performance énergétique français, tout en se percevant comme des «pionniers» du passif, soit d'un modèle reconnu à l'échelle internationale.

Tout d'abord, pour les professionnels concernés, la réglementation française Bepos serait basée sur l'intégration d'une production conséquente d'énergie renouvelable - à partir de technologies telles que les panneaux photovoltaïques - afin de couvrir les besoins énergétiques de la maison. Ceci s'oppose à leur vision de la maison passive privilégiant en premier lieu une efficacité énergétique optimale dans la conception de la maison (bioclimatisme, isolation), laquelle nécessite au final une faible production d'énergie renouvelable pour devenir "positive»:

«On peut faire du Bepos avec un bâtiment RT [2012], même avec une vieille ferme, si on couvre tout en photovoltaïque. Intellectuellement, c'est un gaspillage, ce n'est pas malin, et puis énergétiquement non plus» (entretien, président de la FFCP, Saverne, janvier 2016).

«La RT 2020, qu'est-ce qu'ils vont faire: ils vont rajouter des panneaux solaires sur la RT 2012, ça ne veut pas dire que les maisons seront mieux construites, c'est contre ça qu'on n'est pas d'accord» (entretien, Avenir \& Bois, Salon de l'habitat Strasbourg, avril 2016). 
Deuxièmement, la perception de la «construction durable» et de ses avancées en France, notamment via le cadre législatif, est assez négative, et l'idée que l'« on est en retard ", voire que le cadre réglementaire français ne va pas dans la «bonne direction", revient régulièrement dans les entretiens. Les professionnels prennent alors modèle sur le concept passif allemand et ses appropriations dans d'autres pays à l'échelle européenne et internationale:

«Le Passivhaus, il existe depuis 1983, ça fait quand même plus de 30 ans, c'est de l'autre côté de la frontière, on devrait pouvoir les copier. En plus, la Belgique, l'Autriche, l'Irlande, les États-Unis se sont intéressés au système Passivhaus. [...] Donc, internationalement, ça commence sérieusement à bouger. Le maire de New York a déclaré en juin [2015] aussi que d'ici 5 à 10 ans la ville de New York imposera pour toute construction neuve et toute rénovation lourde les critères du standard passif. Nous, qu'est-ce qu'on fait, les petits Français: on fait notre propre réglementation thermique, franchement c'est grave» (entretien, Agence KMO, Drusenheim, août 2015).

«Nous, comment on entend le passif : c'est le passif du Passivhaus. On en est très, très loin pour la réglementation 2020, la France va appeler ça passif et ça nous embête d'ailleurs un peu parce que ça va galvauder, il y aura une maison passive du PHI, et une maison passive à la française qui n'aura pas du tout la même valeur. Donc je pense que simplement, en France, on est médiocre, on est à la traîne. Pour pouvoir améliorer les statistiques et les apparences, on utilise simplement des mots et on triture les chiffres pour être pas complètement ridicule par rapport aux autres pays européens» (entretien, Maisons Prestige, Horbourg-Wihr, novembre 2015).

C'est souvent en se rendant directement dans des quartiers ou régions de pays voisins ayant opté pour la construction passive, tels que l'Allemagne, l'Autriche ou encore la Suisse, que ces professionnels ont eu une "prise de conscience» et qu'ils se sont lancés dans la démarche de la construction passive:

«On est allés voir un peu ce qui se faisait ailleurs: en Autriche, en Allemagne, pour comprendre comment eux fonctionnaient, parce qu'en France il n'y avait que les prémices du BBC qui étaient là. Et en faisant des formations, [...] mon mari a vu que c'était assez creux, il y avait une démarche mais c'était loin de ce qu'on pouvait faire d'optimal en termes de performance du bâtiment. Et donc on est allé à l'étranger et on a su ce qu'était un bâtiment passif. Et après, pour nous, c'était une évidence qu'on devait aller dans cette voie-là, et donc on s'est spécialisé dans le passif» (entretien, La Maison Innovante, salon de Colmar, mars 2016).

«En 2009, Électricité de Strasbourg avait organisé un voyage d'études en Autriche dans le Vorarlberg, j’y ai été convié et là je suis tombé de ma chaise parce que je me suis rendu compte que l'Autriche est déjà très en avance par rapport à une mise en législation des bâtiments durables, et le Vorarlberg encore plus, ils font depuis maintenant deux ans [2013] : tous les bâtiments neufs ou rénovations lourdes doivent être selon le standard passif, qui remplit à $85 \%$ les critères du Passivhaus» (entretien, Agence KMO, Drusenheim, août 2015).

Suivant un processus d'hybridation des modèles et des référentiels, on observe toutefois que ces constructeurs et maîtres d'œuvre proposent de plus en plus de maisons à énergie positive, en faisant parfois référence à la RT 2020. On peut citer le cas de Maisons Voegelé, qui, en posant des panneaux photovoltaïques sur son modèle de maison passive Chloé, l'a rendu à énergie positive. Le constructeur Maisons Prestige précise également sur son site Internet que "n'importe quelle maison passive peut devenir 
positive, si cela a été prévu au départ. Il suffit de prévoir des fourreaux qui permettront un jour d'installer un dispositif de production d'énergie ${ }^{(55)}$. Nombre d'entre eux annoncent alors construire des maisons "passives et positives».

Cette communication mettant en avant la maison à énergie positive est généralement progressive, tout comme l'a été leur affichage d'une offre de maison passive. Ainsi, on peut constater sur la plaquette de présentation de l'entreprise Avenir \& Bois que la mention «maison à énergie positive» n'est apposée qu'à partir du dernier Salon de l'habitat observé, en 2018. Pour cette entreprise, il y a donc une évolution claire, entre 2013 et 2018, de la maison BBC - qui n'est d'ailleurs pas mentionnée sur la page de garde de la plaquette en 2013 - à la maison passive, jusqu'à la possibilité de la rendre à énergie positive (voir fig. 3). Cette dynamique incrémentale, par paliers, est considérée par les acteurs comme un "progrès» dans l'offre proposée; elle conduit certains maîtres d'œuvre et constructeurs à parler de la construction passive comme de «l'excellence» de la construction, ce qui est à mettre en relation avec la clientèle «haut de gamme» visée dans l'offre ${ }^{(56)}$.

Toutefois, derrière l'affichage et la cohésion apparente de cette offre de construction «alternative» qui acquiert progressivement une valeur normative, les professionnels sont en désaccord sur des points majeurs, qui tiennent à la définition de la maison passive et aux processus de certification permettant d'attester cette performance énergétique.

Fig. 3 : Évolutions de la page de garde des plaquettes d'Avenir \& Bois $(2013,2016,2018)$

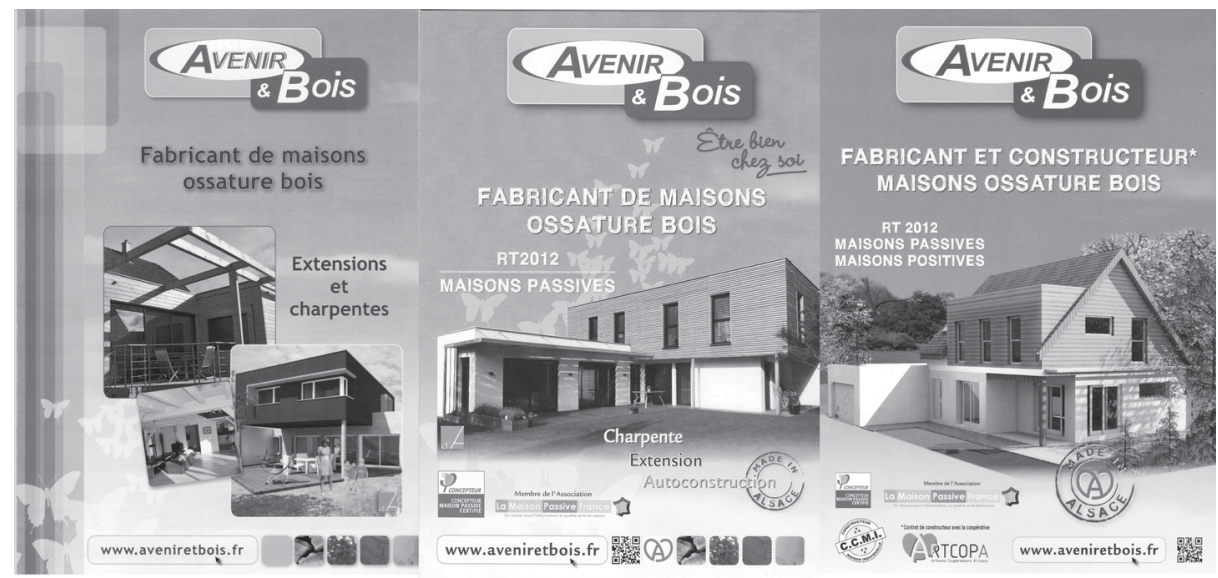

Prospectus recueillis au Salon de l'habitat de Strasbourg $(2013,2016,2018)$

55 maisons-prestige.com/performance/nos-constructions/la-maison-a-energie-positive/.

56 M. Mangold, Pour une sociologie de la «maison durable» (note 1). 


\section{Controverses entre acteurs de la construction locale autour du contenu de la «maison passive»}

Il n'existe pas de définition réglementaire de la maison passive en France, ce qui autorise un certain flou autour de sa spécification et de ses principes concrets. Bien que le standard passif soit basé sur des critères très précis, deux questions clivent les maîtres d'œuvre et les constructeurs: celle du mode de chauffage, revenant à interroger la définition de la maison passive et de son mode de fonctionnement, et celle de la labellisation, opposant à la fois les certifications de la MPF et celle de la FFCP, mais aussi certains concepteurs Passivhaus jugeant la labellisation obligatoire et d'autres l'estimant au contraire non nécessaire.

Premièrement, la maison passive est souvent présentée comme étant une «maison sans chauffage». Cette expression signifie en fait que la maison, par son efficacité énergétique (exposition au soleil via de nombreuses surfaces vitrées au sud, isolation renforcée et système de ventilation double flux récupérant les calories de l'air sortant), peut se passer d'un mode de chauffage dit conventionnel: chaudière, radiateurs, poêle à bois, etc. D'après tous les maîtres d'œuvre et constructeurs rencontrés, il est cependant nécessaire de prévoir ce qui est appelé un «appoint» de chauffage, notamment en cas de période hivernale très peu ensoleillée où la température de la maison chuterait faute d'apports solaires. Il peut alors s'agir de chauffer l'air de la VMC double flux par un système intégré de mini pompe à chaleur, ou encore d'intégrer un ou deux radiateurs électriques avec une production de chauffage limitée (1000 à 2000 watts par exemple), mais suffisante pour obtenir les quelques kWh manquants. Ceci interroge donc tout d'abord la définition du «mode de chauffage» et des modes d'énergie privilégiés. De plus, certains concepteurs de maison passive semblent jouer avec les limites de cette notion de "maison sans chauffage», en intégrant des systèmes de chauffage non apparents puisqu'intégrés dans des plinthes, dans les murs ou encore en chauffage au sol, et fonctionnant de manière automatique:

«Par exemple, ici dans le salon-séjour il y a un plancher qui, lorsqu'on tombe en dessous de la consigne de $21-22^{\circ}$, automatiquement va se mettre en marche et compenser ce que n'apporte pas le soleil, tout simplement et c'est automatique. À l'étage, dans les bureauxchambres, on a un dispositif de plinthes rayonnantes chauffantes qui sont contre le mur, ça fait partie presque de l'esthétique. À l'intérieur, il y a un petit élément chauffant électrique qui rayonne une chaleur douce, donc tout se fait en douceur et est piloté de manière instantanée» (entretien, Maisons Prestige, Horbourg-Wihr, novembre 2015).

«Dans notre système, on a une particularité, on fabrique les murs et à l'usine on va installer des petites nappes chauffantes électriques, [elles] sont dans les murs à $1 \mathrm{~m} 20$, vous ne les voyez pas, il n'y a pas de radiateurs ou d'autre chauffage. On va mettre quelques panneaux photovoltaïques, et ça, ça va permettre de chauffer la maison à plus de $19^{\circ}$, on peut monter jusqu'à $25^{\circ}$. C'est ces panneaux qui vont alimenter ces nappes électriques, donc ils n'auront jamais de facture pour le chauffage» (entretien, Innovalys, Wittenheim, septembre 2015).

D'autres maîtres d'œuvre assument pleinement l'idée de mettre un poêle à bois ou granulés de bois dans la maison, estimant que les particularités climatiques régionales nécessitent un tel «appoint»:

«Oui, il y a un complément, les gens choisissent, c'est soit du poêle bûches, poêle à pellets, un ou deux radiateurs électriques, ou alors des caissettes de préchauffage à la sortie des gaines de VMC double flux: c'est une petite résistance qui va mettre la différence. Chez 
nous, on ne peut pas faire du tout passif sans chauffage, ce n'est pas possible, on a des jours gris, on n'y arrive pas, il faut un appoint. [...] Là, quand il n'y a pas de soleil, c'est le poêle à pellets qui se met automatiquement en marche" (entretien, Avenir \& Bois, salon de Strasbourg, avril 2016).

À l'inverse, pour le président de la FFCP, mettre une pompe à chaleur ou un poêle à bois dans une maison passive est un non-sens, puisqu'il s'agit d'un équipement cô̂teux et produisant trop d'énergie par rapport aux besoins de la maison:

"Mettre un poêle avec un conduit de fumée, étanche à l'air, globalement vous allez mettre 8000 euros, pour quelque chose qui ne sert à rien, c'est débile. Qu'est-ce qu'il se passe si je mets un poêle dans une maison passive? Je vais monter en température à $35^{\circ}$ en 20 minutes, et après qu'est-ce que je fais, j'ouvre la fenêtre? Des moyens démesurés pour des faibles besoins, ça n'a aucun intérêt» (entretien, président de la FFCP, Saverne, janvier 2016).

Toutefois, quel que soit le choix du mode de chauffage, ce dernier ne contraint pas la certification du bâtiment:

«Si demain l'architecte me demande de certifier le bâtiment, s'il y a une chaudière dedans, on le certifiera quand même, on écrira à côté que ce n'est pas nécessaire... Mais ce n'est pas un souci» (ibid.).

Ces questions de chauffage sont avant tout un gage de plus grand confort pour les habitants, questionnant le principe de maison performante énergétiquement dans sa relation concrète aux consommations d'énergie, et ce du côté même des concepteurs. En effet, lorsqu'on relève des températures de confort mentionnées à $21-22^{\circ} \mathrm{C}$, voire $25^{\circ} \mathrm{C}$, on est d'abord loin de la réglementation française stipulant une température de $19^{\circ} \mathrm{C}$ en journée, fixée comme «limite supérieure de température de chauffage [...] dans les locaux à usage d'habitation, d'enseignement, de bureaux ou recevant du public et dans tous autres locaux» ${ }^{(57)}$, mais également au-dessus du calcul du PHPP basé sur la température de $20^{\circ} \mathrm{C}$. Pour le maître d'œuvre suivant, l'intégration d'un mode de chauffage permet en effet de chauffer à la température désirée, sans contrainte, limitant alors les a priori sur une maison passive «froide» en hiver:

«Le problème ne vient pas de l'hiver, on aura largement assez chaud, on a les puissances, on a tout ce qu'il faut, on pourra même chauffer à $25^{\circ} \mathrm{C}$. Bien sûr ça va coûter plus cher mais on peut le faire, on a tout le confort» (entretien, Maisons Prestige, Horbourg-Wihr, novembre 2015).

On perçoit donc que, s'il est de plus en plus question de maison passive à valeur de «modèle», les références effectives ne sont pas stabilisées et peuvent être appropriées différemment selon les professionnels. La question du chauffage n'est pas tranchée et fait intervenir la notion de "confort», se présentant comme primordiale et contrastant fortement avec un discours favorisant les économies de consommation énergétique.

Deuxièmement, la question de la labellisation des maisons divise également les professionnels de la maison passive. L'observation du marché de la construction passive en Alsace sur la période 2013-2018 montre que plusieurs cas de figure sont présents. D’un côté, des concepteurs PHI (diplômés du PHI de Darmstadt) font le plus souvent labelliser Passivhaus - par le PHI - leur première réalisation, mais non les suivantes le coût financier de la certification pour le client est le principal argument invoqué. De 
l'autre, des maîtres d'œuvre ou constructeurs s'affilient à la FFCP et font certifier leur maison passive par cet organisme qui, comme on l'a évoqué, a développé sa propre certification en ayant recours au logiciel PHPP. On trouve également des professionnels affichant une offre de construction passive par leur affiliation à la FFCP mais qui n'ont aucune maison labellisée à leur actif. Le terme de «maison passive » recouvre donc des situations différentes, tout en témoignant bien de l'intérêt commercial de ce référentiel devenu modèle de construction durable.

Dans un cadre de concurrence économique conduisant à une compétition en légitimité, les maîtres d'œuvre formés par le PHI et rattachés à la MPF ont un regard critique sur la certification de la FFCP, et remettent en question le respect du cahier des charges de la «vraie» maison passive et l'évaluation en elle-même:

«Il y a une norme européenne, une formation et un diplôme reconnu, les autres qui s'inventent maison passive, ce n'est pas intéressant» (entretien, Natura Concept, salon de Strasbourg, 2017).

On voit ici apparaître la complexité du processus de certification qui a accompagné l'institutionnalisation de l'éco-construction: des labels et certifications se sont multipliés, souvent élaborés, comme dans le cas de la FFCP, par des acteurs privés. La légitimité d'une définition de la «maison durable» à imposer sur le marché passe aussi par la légitimation de ces labels, lesquels, de manière globale, constituent des formes de gouvernement directes ou indirectes qui poussent - dans une logique concurrentielle et donc de distinction - les acteurs du secteur à faire évoluer leurs procédés de construction et l'efficacité énergétique de leurs maisons:

«La logique de ce gouvernement n'est pas celle de la stabilisation d'un standard qui autorise l'uniformisation des entités et pratiques d'un champ, mais la création continue de labels toujours plus exigeants dont seule une partie des membres d'un champ pourra prétendre s'orner" ${ }^{(58)}$.

Pour les professionnels rattachés à la FFCP, cette certification a l'avantage de permettre d'interagir avec un acteur local et de réaliser des formations à une distance géographique et sur un temps moins contraignant que les formations du PHI, tout en respectant le cahier des charges de la construction passive:

«La MPF, je crois savoir que c'est surtout des constructeurs, des architectes, des maîtres d'œuvre. Dans la Fédération, on a les fournisseurs de matériaux, les fabricants, les bureaux d'études, il me semble que c'est un peu plus large. Et puis la deuxième chose, ce qui me paraissait primordial, c'est que c'est local, c'est à Saverne, en Alsace, et donc pour faire des formations, on a moins de déplacements, on est à proximité» (entretien, Maisons Prestige, Horbourg-Wihr, novembre 2015).

«Là on habite aussi à $10 \mathrm{~km}$ du siège de la Fédération, donc ça nous semblait assez évident d'avoir ce côté proximité quoi. Après, je pense que l'intelligence voudrait d'être dans les deux pour que les clients puissent se retrouver dans les deux plateformes» (entretien, $L a$ Maison Innovante, Colmar, mars 2016).

De fait, la FFCP met en avant le fait d'aller «plus loin» que la certification du PHI en intégrant notamment des critères d'évaluation sur la qualité de l'air : 
«Nous on certifie bien plus de choses, on vérifie en permanence la qualité de l'air intérieur, typiquement le taux de $\mathrm{CO}_{2}$, on garantit une performance énergétique, donc on certifie un bâtiment sur un an et au bout d'un an on valide le certificat. [...] Donc performance énergétique comme le PHI, qualité de l'air intérieur, pérennité des parois, on ne vérifie pas uniquement le critère énergétique ou on risque d'arriver à des chantiers loupés qui fonctionnent pas» (entretien, président de la FFCP, Saverne, janvier 2016).

Cependant, la communication internationale autour de la maison passive aurait tendance à invisibiliser le travail de la FFCP. En effet, si on veut réaliser un état des lieux de la construction de maisons passives en France, on s'aperçoit que seules les maisons ayant été certifiées par la MPF - reconnue comme organisme habilité à délivrer le label - et donc par le PHI sont intégrées dans le catalogue des maisons passives disponible sur le site Passivhausprojekte. En Alsace, on ne dénombre alors début 2019 que 13 maisons dans la base de données ${ }^{(59)}$, ce qui pose problème aux maîtres d'œuvre et constructeurs de maison passive rattachés à la FFCP :

«L'inconvénient, c'est que la MPF représente officiellement le PHI et donc les maisons certifiées par la MPF remontent sur le site du PHI, alors que nous, ceux de la Fédé ne remontent pas, c'est un label propre, mais qui est basé sur les mêmes exigences que le PHI» (entretien, Maisons Prestige, Horbourg-Wihr, novembre 2015).

Les controverses professionnelles à l'œuvre dans le développement d'un marché de la «maison durable» se retrouvent dans la définition même d'un modèle de «maison passive». Les professionnels peuvent aussi jouer du flou et l'alimenter, pour certains, en même temps que se développe (et ce d'autant plus qu'il n'y a pas de cadre précis) un marché, de façon similaire à l'analyse du «succès du flou» du développement durable ${ }^{(60)}$.

\section{Conclusion}

L'analyse de la diffusion du référentiel Passivhaus et de ses appropriations par les acteurs de la construction en Alsace illustre nettement la circulation de modèles de durabilité et les questions d'échelle en développement durable. La certification Passivhaus a été développée dans un cadre territorial précis, à Darmstadt en Allemagne, avant de devenir un outil de calcul et un référentiel adaptable dans divers contextes territoriaux, énergétiques et climatiques. La référence à la construction passive s'affranchit cependant, dans d'autres contextes territoriaux, de l'organisme de référence du Passivhaus Institut, à partir du développement de certifications alternatives qui peuvent consister à redéfinir certains seuils et objectifs énergétiques de la certification Passivhaus de départ. Les acteurs politiques et privés de la construction se réapproprient donc le référentiel pour l'ajuster à leurs propres conditions de faisabilité.

En Alsace, on a vu que l'offre de construction de maison passive s'est amplement développée durant la décennie actuelle, et la référence au Passivhaus a fait l'objet de réappropriations suscitant des controverses entre professionnels. L'opportunité commerciale de renouvellement que la construction passive a offert sur le marché de la maison individuelle explique en partie le développement rapide et les appropriations

59 www.passivhausprojekte.de/.

60 P. Hamman, Sociologie urbaine (note 4), p. 29. 
diverses qu'en font les maîtres d'œuvre et constructeurs en l'absence de cadre normatif français sur la construction passive. Les acteurs enquêtés se placent dans la position de pionniers ou d'«innovateurs» et mobilisent pour cela le référentiel de la durabilité tout en proposant des contenus constructifs variables, notamment sur les questions de chauffage dans une maison passive, où des équipements intégrés pour le confort thermique des habitants sont «verdis» (énergie renouvelable) alors que l'objectif de départ d'une maison passive est de limiter la production d'énergie au maximum.

$\mathrm{Au}$ final, on peut tirer trois enseignements principaux de l'étude du cas de la construction passive dans la circulation de modèles de durabilité :

1. Elle donne à voir le travail de production d'une offre qui se veut exemplaire et innovante, toujours en train de se faire et encore soumise à controverse au sein même du corps professionnel. C'est là une analyse courante des projets territorialisés en développement durable dont l'aspect concret et substantiel ne coïncide pas pleinement avec l'aspect procédural et les modes d'énonciation des objectifs génériques de la durabilité. À l'intersection des sciences de la technique, sciences de la nature et sciences humaines, le logement constitue un objet des humanités environnementales et doit aujourd'hui articuler objectifs environnementaux et modes d'habiter ${ }^{(61)}$.

2. À partir de cette circulation de références à valeur d'exemplarité, on perçoit également la concurrence entre différents modèles de construction durable et l'approche énergétique qui domine actuellement cette production. En ce sens, la construction passive se situe dans la suite logique de politiques environnementales visant la «transition énergétique», concrétisées à travers la production de réglementations thermiques. Bien que la «transition énergétique » ait connu une trajectoire différente entre la France et l'Allemagne ${ }^{(62)}$, on saisit ici une certaine convergence entre les deux pays dans cette approche énergétique de la construction durable.

3. Sur la question des échelles du développement durable, la référence au Passivhaus en Alsace illustre des logiques de circulation de savoirs entre l'Allemagne et la France, dans le cadre d'un territoire propice aux échanges transfrontaliers. Toutefois, il semble que les acteurs de la construction alsaciens soient particulièrement intéressés par la dimension internationale prise par ce modèle de durabilité, et non uniquement son application dans un espace binational de proximité. De plus, on a pu constater que la construction passive a d'abord été développée en France à travers des salons nationaux de la construction (à Grenoble et Paris) avant de se développer sur le marché local de la construction en Alsace. Le cas de la FFCP, acteur se déclarant comme national mais agissant en grande partie à l'échelle régionale, illustre ces jeux d'échelles dans le développement de la construction durable avec des références à la fois territorialisées, nationales et internationales.

61 Nicole Mathieu, "Modes d'habiter", "cultures de la nature": des concepts indissociables", in: Aurélie Choné, Isabelle Hajex et Philippe Hamman (dir.), Guide des Humanités environnementales, Villeneuve d'Ascq, Presses universitaires du Septentrion, 2016, p. 567-581.

62 Guillaume Christen, Philippe Hamman, Mathias Jehling et Maurice Wintz (dir.), Systèmes énergétiques renouvelables en France et en Allemagne. Synergies et divergences, Paris, Éditions Orizons, 2014. 


\section{Résumé}

Cet article interroge la circulation de modèles et de savoirs sur la durabilité entre Allemagne et France à partir de l'exemple du référentiel Passivhaus. Cette certification se référant à une construction dite durable, développée en Allemagne au début des années 1980, se retrouve depuis une dizaine d'années en France à travers le développement et la structuration d'une offre de construction passive. Dans un premier temps est exposé le contexte de diffusion de ce référentiel, devenu modèle à l'échelle internationale. Puis, dans un second temps, la contribution livre une analyse des appropriations dont il fait l'objet à l'échelle locale, à partir d'enquêtes de terrain menées sur le marché de la maison individuelle en Alsace entre 2013 et 2018. Les jeux d'acteurs et controverses autour du contenu de la maison passive offrent alors, dans une troisième section, une lecture des échelles et des circulations de modèles de durabilité entre Allemagne et France, où la dimension internationale vaut comme référence locale de légitimation.

\section{Zusammenfassung}

Dieser Artikel untersucht am Beispiel des Energiestandards Passivhaus, wie sich Modelle und Wissen über die Nachhaltigkeit zwischen Deutschland und Frankreich verbreiten. Diese Zertifizierung bezieht sich auf das nachhaltige Bauen, das in den 1980er Jahren in Deutschland entwickelt wurde und seit zehn Jahren in Frankreich Fuß fasst. Untersucht werden zunächst die Verbreitungswege dieses Standards, der heute auf internationaler Ebene als Modell dient. In einem zweiten Schritt wird dessen Anwendung auf lokaler Ebene analysiert, ausgehend von Felduntersuchungen, die zwischen 2013 und 2018 auf dem elsässischen Markt für Passivhäuser durchgeführt wurden. Zuletzt werden die Kontroversen über die wahren Anforderungen eines Passivhauses erwähnt. Hier scheint die internationale Anerkennung des Standards einen besonderen Legitimationseffekt auflokaler Ebene zu bewirken. 